\title{
Evaluación del uso de la microalga Chlorella vulgaris como Biorremediadora de vertimientos de la Industria Acuícola en el Caribe Colombiano.
}

\section{Evaluation of the use of the microalgae Chlorella vulgaris as a Biorremediator of discharges from the Aquaculture Industry in the Colombian Caribbean.}

\author{
Ildefonso Baldiris Navarro ${ }^{1}$ Martha Torres Virviesca ${ }^{2}$ Jorge Sánchez Aponte $^{1}$ \\ ${ }^{1}$ SENA - Centro Internacional Náutico, Fluvial y Portuario Cartagena, Colombia * \\ 2 Universidad del Sinú
}

Recibido: 15/feb/2019 Revisado: 30/abr/2019

Aceptado: 30/may/2019 Publicado: 30/jul/2019

Resumen Los vertimientos de las industrias acuícolas, impactan ambientalmente los cuerpos de aguas naturales por las inadecuadas prácticas de manejo, sobre todo alimentación y fertilización de los sistemas de cultivo, alcanzado a tener un efecto eutrofizador, debido al aumento de nutrientes principalmente de fósforo y nitrógeno, que pueden ser contaminantes peligrosos reduciendo el oxígeno en el agua. Los nutrientes del agua ( $\mathrm{P}-\mathrm{N})$ pueden eliminarse de los efluentes acuíferos por procesos fisicoquímicos convencionales que son muy complejos y costosos. En esta investigación, se evaluó la capacidad de biodegradación de estos nutrientes, utilizando la microalga Chlorella vulgaris. Los bioensayos consistieron en cuatro combinaciones de agua residual-microalga al 10\%, 30\%, 50\%, $70 \%$ y una solución de control sin contaminante; en los resultados se observa que la mayor remoción de DQO se obtuvó en la concentración del $10 \%$ con un $57.7 \%$ de remoción y la menor eliminación en la del $50 \%$ que fue menor al $1 \%$. Los ensayos al $30 \%$ y $70 \%$ removieron $48.8 \%$ y $43.8 \%$ respectivamente. Se concluye que la microalga Chlorella vulgaris, ayuda en la remoción de DQO de aguas residuales con alta carga de nutrientes

Palabras claves: chlorella vulgaris; industria acuícola; DQO.

\begin{abstract}
Effluents from aquaculture industries have an environmental impact on natural water bodies due to inadequate management practices, especially feeding and fertilization of crop systems, which have had a eutrophication effect, due to the increase of nutrients mainly of phosphorus $(\mathrm{P})$ and nitrogen $(\mathrm{N})$, which might be dangerous pollutants that reduce oxygen in water. Water nutrients (P-N) may be removed from aquifer effluents by conventional physicochemical processes that are very complex and expensive. In this research, the biodegradability of these nutrients was evaluated using the microalgae Chlorella vulgaris. The bioassays consisted of four $10 \%, 30 \%, 50 \%, 70 \%$ microalgae-wastewater combinations and a control solution; Results showed that the highest COD removal was obtained at the $10 \%$ concentration with $57.7 \%$ remotion and the lowest elimination at the $50 \%$ which was lower than $1 \%$. Bioassays at $30 \%$ and $70 \%$ removed $48.8 \%$ and $43.8 \%$ respectively. It was concluded that the microalgae Chlorella vulgaris, may help in the removal of COD of wastewater with high nutrient load.
\end{abstract}

Keywords: Chlorella vulgaris, aquaculture industries, COD.

Teknos Revista Científica | Volumen 19 No.1 - Julio 2019 | ISSN 1900-7388 (papel) | ISSN 2539-2190 (digital) DOI: https://doi.org/10.25044/issn.2539-2190 


\section{Introducción}

La calidad de las fuentes hidricas en los últimos años ha estado bajo una gran presión antropogénica, debido al crecimiento de la población, la industrialización y el uso de malas prácticas agropecuarias, lo que ha traído consigo efluentes cargados de contaminantes, siendo el fósforo y nitrógeno los más comunes. Una de las fuentes frecuentes de estos nutrientes, es la agroindustria y en especial la industria camaronera.

La industria acuícola a nivel mundial, es una de las que presenta mayor crecimiento en el sector alimenticio, con un aumento anual promedio de 5.8\% (Piracha, 2015). Con la expansión e intensificación de las fincas camaroneras continentales que disponen sus vertimientos cargados con altas concentraciones de nutrientes sin ningún tipo de tratamiento previo, se podría llegar a un nivel de eutrofización del sistema acuático y degradar hábitats bentónicos y pelágicos $(\mathrm{Li}$ et al., 2019).

La biorremediación es actualmente una técnica sostenible para eliminar diversos contaminantes de los ecosistemas, ya que además de su bajo costo, tiene una alta eficiencia para la remoción de nutrientes orgánicos e inorgánicos y no produce subproductos nocivos para el ambiente. Durante los procesos de biorremediación el carbono, el nitrógeno, el fósforo y otros elementos, son asimilados por la microalga para su metabolismo y crecimiento celular, produciendo asi un efluente con mejor calidad. Las microalgas comienzan a jugar un papel cada vez más importante en el tratamiento del agua contaminada con compuestos orgánicos y fracciones de petróleo. Se ha demostrado que microalgas como, Prototheca zopfii, Selenastrum capricornutum, Scenedesmus acutus y Ankistrodesmus braunii, Chlamydomonas ulvaensis, Chlorella pyrenoidosa y Scenedesmus brasiliensis, y el fitoflagelado Euglena gracilis, degradan de manera eficiente diversos compuestos orgánicos, incluidos derivados del petróleo.
Estudios recientes han demostrado la aplicación eficiente de las microalgas en el tratamiento de agua residual de porquerizas (Wang, Xiong, Hui, \& Zeng, 2012), efluentes de procesadoras de palma africana (Emparan, Jye, Danquah, \& Harun, 2020)(Khalid, Yaakob, Abdullah, \& Takriff, 2019), municipales (Ling et al., 2019)(Hernández-García et al., 2019), procesadoras de vinos (Ganeshkumar et al., 2018) y lixiviados (Nair, Senthilnathan, \& Nagendra, 2019)(Hernández-García et al., 2019), entre otros.

En este estudio se evaluó la capacidad de la microalga Chlorella vulgaris para biorremediar el agua residual de una industria acuícola y el efecto que ésta tiene sobre el crecimiento de la microalga.

\section{Materiales y métodos}

La microalga Chlorella vulgaris se obtuvo de la Colección Cultural de Algas en SENA - Centro Internacional Náutico Fluvial y Portuario, ubicado en la ciudad de Cartagena de Indias, Colombia, que desde el año 2016, viene trabajando el campo de la biorremediación con microalgas (Baldiris et al., 2016).

\subsection{Reactivos}

El medio Conway (Tompkins et al.,1995) modificado por Virviescas \& Aponte 2016, se utilizó en el laboratorio de biotecnología de Centro Internacional Náutico Fluvial y Portuario SENA con la siguiente composición: $\mathrm{FeCl} 3 \cdot 6 \mathrm{H} 2 \mathrm{O}(26 \mathrm{~g}), \mathrm{MnCl} 2 \cdot 4 \mathrm{H} 2 \mathrm{O}(0,72$ g), H3BO3 (67,2 g), EDTA (90 g), Na2HPO4.12H2O (40 g), NaNO3 (200 g), Na2SiO3 (40 g), H2O (2 L), trazas de solución de metal $(2 \mathrm{ml})$ y Decamil B12 (100 $\mathrm{ml})$.

La solución de trazas metálicas que fue usada, estaba compuesta de $\mathrm{ZnCl} 2(2,1 \mathrm{~g}), \mathrm{CoCl} 2 \cdot 6 \mathrm{H} 2 \mathrm{O}(2 \mathrm{~g})$, $(\mathrm{NH} 4) 6 \mathrm{Mo} 7 \mathrm{O} 24 \cdot 4 \mathrm{H} 2 \mathrm{O}(0.9 \mathrm{~g}), \mathrm{CuSO} 4.5 \mathrm{H} 2 \mathrm{O}(2 \mathrm{~g})$ y agua destilada $(100 \mathrm{ml})$, la solución de vitaminas estaba compuesta de Decamil (210 mg) y agua destilada (100 ml). (Baldiris, 2017). 


\subsection{Sistemas y métodos de cultivo}

Las microalgas se cultivaron usando un sistema de escalado por lotes, comenzando desde una placa de Petri, hasta un tubo de ensayo, luego a un Matraz Erlenmeyer de $250 \mathrm{ml}$ y finalmente a un Matraz Erlenmeyer de $1000 \mathrm{ml}$. Las cepas se mantuvieron en medio de cultivo Conway modificado. Las condiciones de cultivo incluyen una temperatura de $24 \pm 2^{\circ} \mathrm{C}$, lámparas fluorescentes de $39 \mathrm{~W}$ como fuente de iluminación artificial, con irradiación de 5000 lux, fotoperiodo de 12 horas de luz y 12 de oscuridad, aireación de $0.7 \mathrm{vvm}$ usando aire atmosférico a través de un sistema mecánico de ventilación sin inyección de $\mathrm{CO} 2$. Cada mezcla de microalga con agua residual se cultivó dos veces durante el tiempo necesario, para lograr la reducción de la concentración del agua residual de las piscinas camaroneras.

El crecimiento de la biomasa se analizó a intervalos iguales de $24 \mathrm{~h}$ por espectrofotometría a $680 \mathrm{~nm}$ y conteo en cámara newbauer, luego de estos se encontró una relación entre las variables por regresión lineal. Para analizar la variación del crecimiento de la microalga en el agua residual, se puso en contacto con soluciones de agua residual al $10 \%, 30 \%, 50 \%$ y $70 \%$ y se dejó interactuar.

La velocidad específica de crecimiento se calculó de la siguiente manera:

$$
\mu\left(d i a^{-1}\right)=\frac{\left(\ln X_{2}-\ln X_{1}\right)}{\left(t_{2}-t_{1}\right)}
$$

El porcentaje de inhibición se calculó de acuerdo a la siguiente ecuación:

$$
\% \text { Inhibición }=\left[\frac{D . O_{c}-D \cdot O_{m}}{D . O_{c}}\right] \times 100
$$

La demanda química de oxígeno (DQO) se define como la cantidad de oxidante especificado que reacciona con una muestra bajo condiciones controladas. La DQO a menudo se usa como una medida de contaminantes en aguas residuales y aguas naturales. En esta investigación se utilizó el método de reflujo cerrado, el cual es más económico y produce menos residuos líquidos peligrosos (APHA, American Public Health Association, 2012). La remoción de $\mathrm{DQO}$ se evaluó por medio de la expresión:

$$
\% \text { Remoción }=\left[\frac{C_{i}-C_{f}}{C_{i}}\right] \times 100
$$

Para esta prueba fueron usados; agua ultrapura, dicromato de potasio ( $\mathrm{K} 2 \mathrm{Cr} 2 \mathrm{O} 7)$ al $99.5 \%$, ácido sulfúrico $(\mathrm{H} 2 \mathrm{SO} 4)$, sulfato de mercurio $(\mathrm{HgSO} 4)$ grado reactivo, biftalato de potasio (HOOCC6H4COOK), sulfato ferroso amoniacal hexahidratado ( $\mathrm{Fe}(\mathrm{NH} 4) 2(\mathrm{SO} 4) 2 \cdot 6 \mathrm{H} 2 \mathrm{O}), \quad 1,10 \quad$ fenantrolina monohidratada y sulfato ferroso heptahidratado (FeSO4-7H2O).

\section{Resultados}

\subsection{Crecimiento celular de la microalga}

La densidad celular fue medida diariamente por un período de 10 días, para encontrar su relación con la densidad óptica. El resultado puede observarse en la figura 1, donde se aprecia una relación lineal entre las variables, al hacer el análisis estadístico se encontró un p-valor igual a 0.0001 lo que confirma esta relación. También se encontró un coeficiente de correlación (R) de 0.92 y un R2 (ajustado) de $83 \%$ y un estadístico de Durbin-Watson de 2.92.

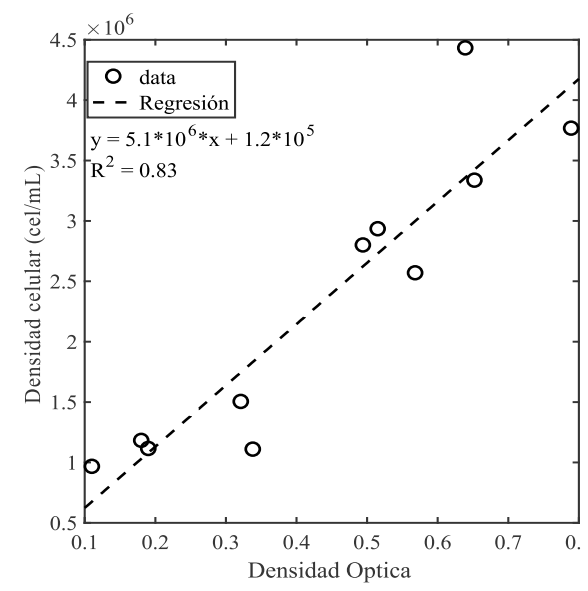

Figura 1. Regresión lineal entre densidad óptica y densidad celular para Chlorella vulgaris. 
El modelo matemático ajustado para los datos experimentales es:

$$
\text { D. } C=5.1 \times 10^{6} \times D . O+1.2 \times 10^{5}
$$

Donde D.C es la densidad celular en cel $/ \mathrm{mL}$ y DO es la densidad óptica medida a $680 \mathrm{~nm}$. Con este modelo se midió la absorbancia y se obtuvieron datos de la densidad celular.

El estadístico R2 indica que el modelo ajustado explica un $83 \%$ de la variación en la población (ver figura1).

La figura 2 muestra los resultados obtenidos del crecimiento de la microalga Chlorella vulgaris en diferentes concentraciones de agua residual de industrias acuícolas. En la gráfica se puede observar que se presentó una inhibición en el crecimiento de la microalga, ya que el crecimiento estuvo por debajo de la muestra de control sin agua residual.

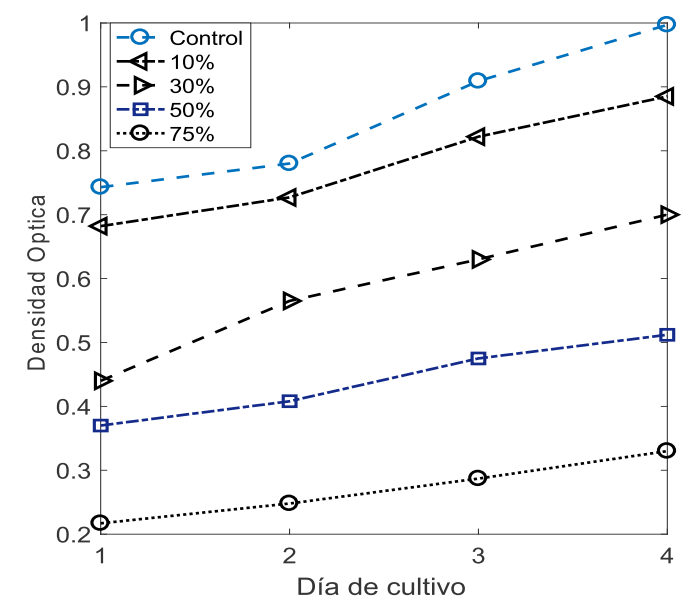

Figura 2. Crecimiento de la microalga en agua residual de camaroneras

Realizando un análisis anova de los diferentes bioensayos, se puede apreciar en la figura 3 que el tratamiento de control y el bioensayo al $10 \%$, no presentan diferencia significativa en sus resultados, mientras que los demás bioensayos si presentan diferencias significativas con el ensayo de control. Esto puede indicar que bioensayos posteriores, deben hacerse a máximo un $10 \%$ de dilución en pos de conseguir un nivel aceptable de crecimiento o en su defecto aplicar nutrientes que beneficien el crecimiento de la microalga en los bioensayos al $30 \%$, $50 \%$ y $70 \%$.

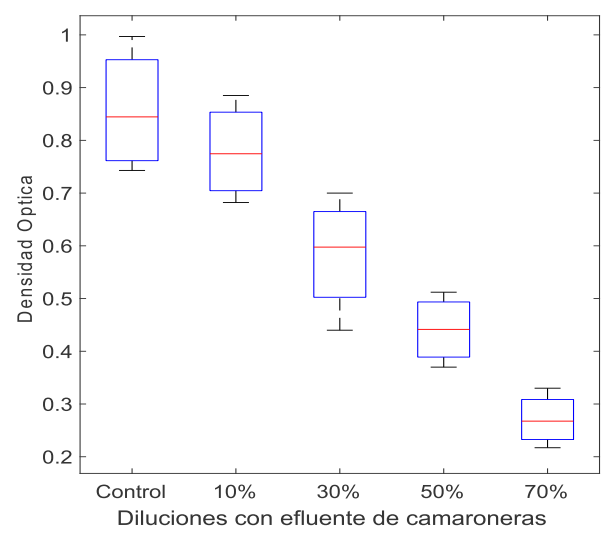

Figura3. Comparación de crecimiento en bioensayos

\subsection{Velocidad especifica de crecimiento e inhibición de cultivos}

Para los diferentes bioensayos se encontró que la velocidad específica de crecimiento, no presentó variación significativa entre ellos. La microalga se adaptó al medio y pudo crecer en el agua residual, aunque por debajo del blanco. La inhibición presentó niveles altos en los bioensayos variando de $11.23 \%$ hasta $66.90 \%$. El bioensayo que presentó mayor inhibición fue el de $70 \%$ de dilución; en este a pesar de que la microalga creció, estuvo muy por debajo del control.
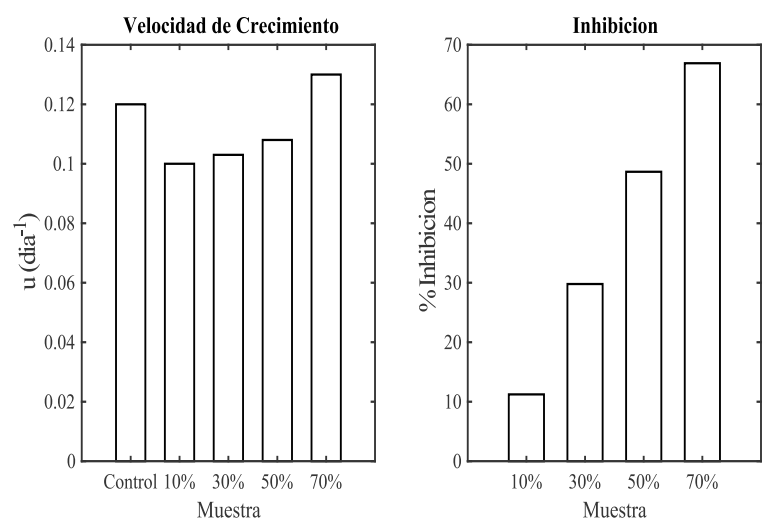

Figura 4. Velocidad de crecimiento y porcentajes de inhibición para bioensayos 
Esto quiere decir que altas concentraciones de agua de industrias acuícolas, no apoyan el crecimiento celular y las microalgas son inhibidas, como puede apreciarse en los bioensayos. Este fenómeno puede ser causado por el color oscuro que posee el vertimiento, el cual puede bloquear la penetración de la luz al cultivo (Cheah, Show, Juan, Chang, \& Ling, 2018). La baja producción de biomasa en los bioensayos con agua residual, sugieren la necesidad de suplementar los cultivos de microalgas con nutrientes para alcanzar los niveles obtenidos con solo medio de cultivo, esto puede ser causado por la baja concentración de nutrientes especialmente $\mathrm{NH} 4+-\mathrm{N}$, NO2--N y NO3--N (Kuo et al., 2016). La presencia de Protozoa puede ser también una causa de baja producción de biomasa, ya que éstos se alimentan de la microalga, lo que puede hacer colapsar el cultivo (Tejido-Nuñez, Aymerich, Sancho, \& Refardt, 2019).

\subsection{Demanda química de oxígeno}

Las sustancias orgánicas e inorgánicas oxidables presentes en la muestra de microalgas con agua agroindustrial, se oxidan mediante reflujo cerrado en solución fuertemente ácida, con un exceso de dicromato de potasio en presencia de sulfato de plata, que actúa como agente catalizador y de sulfato mercúrico adicionado para eliminar la interferencia de los cloruros.

Los análisis realizados a los diferentes bioensayos, estan esquematizados en la figura 5 .

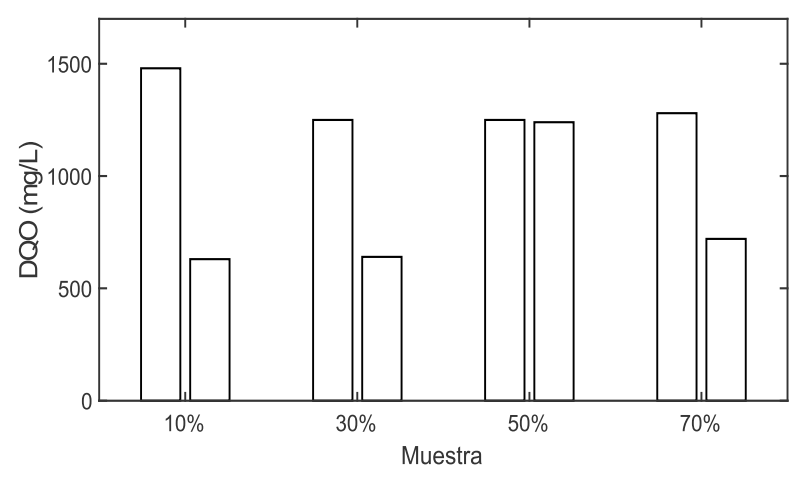

Figura 5. Remoción de DQO en bioensayos
Puede observarse que la mayor remoción de DQO se obtuvó en el bioensayo al $10 \%$ con un $57.7 \%$ de remoción y la menor remoción en el ensayo al $50 \%$ que fue menor al $1 \%$. Los ensayos al $30 \%$ y $70 \%$ removieron $48.8 \%$ y $43.8 \%$ respectivamente.

\section{Conclusión}

Se evidenció que la microalga Chlorella vulgaris, ayuda en la remoción de DQO de aguas residuales con alta carga de nutrientes, aunque presentó una inhibición en todas las concentraciones de los bioensayos, ya que el crecimiento celular nunca fue mayor a la muestra de control sin agua residual.

\section{Referencias}

APHA, American Public Health Association, A. - A. W. W. A. (2012). Standard methods for the examination of water and wastewater (21st ed.). United states of america.

Baldiris (2017) "Validation, characterization and comparison of microalgae Chlorella vulgaris and Chlamydomona reinhardtii growth kinetics"Chemtech J, vol. 10, no. 15, 411-420.

Cheah, W. Y., Show, P. L., Juan, J. C., Chang, J. S., \& Ling, T. C. (2018). Microalgae cultivation in palm oil mill effluent (POME) for lipid production and pollutants removal. Energy Conversion and Management, 174(August), 430-438.

https://doi.org/10.1016/j.enconman.2018.08.057

Emparan, Q., Jye, Y. S., Danquah, M. K., \& Harun, R. (2020). Cultivation of Nannochloropsis sp. microalgae in palm oil mill effluent (POME) media for phycoremediation and biomass production: Effect of microalgae cells with and without beads. Journal of Water Process Engineering, 33(October 2019), 101043. https://doi.org/10.1016/j.jwpe.2019.101043

Ganeshkumar, V., Subashchandrabose, S. R., Dharmarajan, R., Venkateswarlu, K., Naidu, R., \& Megharaj, M. (2018). Use of mixed wastewaters from piggery and winery for nutrient removal and lipid production by Chlorella sp. MM3. Bioresource Technology, 256(February), 254-258.

https://doi.org/10.1016/j.biortech.2018.02.025

Hernández-García, A., Velásquez-Orta, S. B., Novelo, E., Yáñez-Noguez, I., Monje-Ramírez, I., \& Orta Ledesma, M. T. (2019). Wastewater-leachate treatment by microalgae: Biomass, carbohydrate and lipid production. Ecotoxicology and Environmental Safety, 
174(August 2018), 435-444.

https://doi.org/10.1016/j.ecoenv.2019.02.052

Khalid, A. A. H., Yaakob, Z., Abdullah, S. R. S., \& Takriff, M. S. (2019). Assessing the feasibility of microalgae cultivation in agricultural wastewater: The nutrient characteristics. Environmental Technology and Innovation, 15, 100402.

https://doi.org/10.1016/j.eti.2019.100402

Kuo, C. M., Jian, J. F., Lin, T. H., Chang, Y. Bin, Wan, X. H., Lai, J. T., ... Lin, C. S. (2016). Simultaneous microalgal biomass production and $\mathrm{CO} 2$ fixation by cultivating Chlorella sp. GD with aquaculture wastewater and boiler flue gas. Bioresource Technology, 221(75), 241-250.

https://doi.org/10.1016/j.biortech.2016.09.014

Li, M., Callier, M. D., Blancheton, J. P., Galès, A., Nahon, S., Triplet, S., ... Roque d'orbcastel, E. (2019). Bioremediation of fishpond effluent and production of microalgae for an oyster farm in an innovative recirculating integrated multi-trophic aquaculture system. Aquaculture, 504(April 2018), 314-325. https://doi.org/10.1016/j.aquaculture.2019.02.013

Ling, Y., Sun, L. ping, Wang, S. ying, Lin, C. S. K., Sun, Z., \& Zhou, Z. gang. (2019). Cultivation of oleaginous microalga Scenedesmus obliquus coupled with wastewater treatment for enhanced biomass and lipid production. Biochemical Engineering Journal, 148(May), 162-169.

https://doi.org/10.1016/j.bej.2019.05.012

Nair, A. T., Senthilnathan, J., \& Nagendra, S. M. S. (2019). Application of the phycoremediation process for tertiary treatment of landfill leachate and carbon dioxide mitigation. Journal of Water Process Engineering, 28(February), 322-330.

https://doi.org/10.1016/j.jwpe.2019.02.017

Piracha, A. (2015). EDevelopment-assessment as "smart eplanning" for New South Wales (NSW) Australia. CUPUM 2015 - 14th International Conference on Computers in Urban Planning and Urban Management.

Tejido-Nuñez, Y., Aymerich, E., Sancho, L., \& Refardt, D. (2019). Treatment of aquaculture effluent with Chlorella vulgaris and Tetradesmus obliquus: The effect of pretreatment on microalgae growth and nutrient removal efficiency. Ecological Engineering, 136(May), 1-9. https://doi.org/10.1016/j.ecoleng.2019.05.021

Tompkins J., Deville M.M., Day J.G. \& Turner M.F. (1995). Catalogue of Strains. Titus Wilson and Son Ltd. Kendall. U.K. 166 pp.
Virviescas, M. J. T., \& Aponte, J. H. S. (2016). Avances del Banco de Cepas de Microalgas en el Centro Internacional Náutico Fluvial y Portuario del SENA Advances on Microalgae Strains Bank at Centro International Náutico Fluvial y Portuario of SENA. Revista Sennova: Revista del Sistema de Ciencia, Tecnología e Innovación, 2(1), 30-41.

Wang, H., Xiong, H., Hui, Z., \& Zeng, X. (2012). Mixotrophic cultivation of Chlorella pyrenoidosa with diluted primary piggery wastewater to produce lipids. Bioresource Technology, 104, 215-220. https://doi.org/10.1016/j.biortech.2011.11.020 